Licenciada en Literatura Dramática y Teatro por la UNAM. Actualmente estudia la licenciatura en Etnohistoria en la Escuela Nacional de Antropología e Historia (ENAH) y la maestría en Estudios Mesoamericanos. Se ha dedicado a la docencia de la Literatura en nivel medio-superior, impartiendo asimismo talleres para profesores de arte. En la práctica teatral, realiza montajes como actriz desde hace doce años y como productora desde hace cuatro años. Ha presentado diversas ponencias sobre teatro $y$ etnohistoria en México y Brasil.

\title{
EL JUEGO DE LA CONQUISTA DE SAN AGUSTÍN TLACOTEPEC (OAXACA. MÉXICO)
}

\author{
KARINA CASTRO SANTANA
}

\section{RESUMEN}

Desde que llegaron las danzas de conquista a territorios mexicanos, éstas se han constituido en más que una tradición: se trata de un medio de expresión para las comunidades indígenas que aún desean mostrar su cultura y su punto de vista respecto a un hecho que fue trascendental en la historia de México, así como asegurar y preservar una identidad colectiva.

Trataremos de llevar a cabo en este trabajo la descripción y análisis de la celebración de una de estas danzas que se realiza cada 28 de agosto, en la Mixteca Alta del estado de Oaxaca (México), concretamente en la comunidad indígena de San Agustín Tlacotepec. Dicha festividad integra elementos ceremoniales y dancísticos junto a la conmemoración propia de la onomástica del doctor y obispo de Hipona.

\section{THE GAME OF CONQUEST IN SAN AGUSTÍN TLACOTEPEC (OAXACA, MÉXICO)}

\section{ABSTRACT}

Since the conquest dances arrived on Mexican soil they bave become much more than a just a tradition. They are a form of expression for indigenous communities who still wish to demonstrate their culture and point of view with regards to a transcendental event in the history of Mexico, not to mention ensuring and preserving a collective identity.

This work will provide a description and analysis of one of these dance celebrations which takes place in the indigenous community of San Augustin Tlacotepec every $28^{\text {th }}$ August in Mixteca Alta in the state of Oaxaca (Mexico). This festivity integrates ceremonial and dance elements alongside the saint's day commemoration of the doctor and bishop of Hipona.

Actualmente en México se realizan numerosas fiestas patronales en las que se pueden observar diversos elementos teatrales que contribuyen a la efectividad de estos festejos. Dichas celebraciones se llevan a cabo cada año, principalmente en las provincias del país; debido a que las comunidades adquirieron, durante el virreinato novohispano, el nombre de algún patrono católico, acompañado de su segundo nombre autóctono. Sin embargo, se respeta el calendario católico para solemnizar sus ceremonias. 
En este artículo se presenta la descripción y análisis de una celebración que se practica en la Mixteca Alta del estado de Oaxaca, en el sur de México. En la comunidad indígena de San Agustín Tlacotepec, se lleva a cabo anualmente, el 28 de agosto, una festividad en la que se conmemora el aniversario luctuoso de San Agustín doctor y obispo. Este festejo integra entre sus elementos ceremoniales, de culto al santo, un juego de conquista.

El término «juego» es aplicado sobre todo a las danzas-drama, debido a que estas representaciones tienen como connotación la de romper con la vida cotidiana de la comunidad en la que se lleva a cabo. «Durante las fiestas [...] el tiempo normal se detiene y da paso al tiempo sagrado» explica Lilian Scheffler, quien a su vez recuerda las palabras de Huizinga:

Esta cancelación temporal del mundo cotidiano se presenta ya de plano en la vida infantil; pero también la vemos claramente en los grandes juegos arraigados en el culto de los pueblos primitivos... Esta suspensión temporal de la vida social ordinaria en gracia a un tiempo sagrado de juego, la podemos encontrar también en culturas avanzadas ${ }^{1}$.

El juego de San Agustín Tlacotepec consiste en la ejecución bailada y dialogada de varios pasajes de la conquista de México en 1521. Estos episodios son presentados en orden cronológico a lo largo de dos días en los que se narra desde la llegada de los españoles, hasta la derrota del pueblo azteca. A pesar de que el primer registro de una teatralización de la conquista en México data del año $1566^{2}$, esta representación tiene su origen en el año de 1932, cuando el señor Isidro Sánchez, habitante de Tlacotepec, adquirió el libreto de la danza en las costas del estado vecino de Guerrero.

La organización del juego comienza unos meses antes, cuando los mayordomos de la fiesta eligen al encargado de dirigirlo ${ }^{3}$. La conformación del elenco se lleva a cabo por decisión del director: él debe ir a solicitar a cada uno de los participantes que se incorpore a la danza; tal solicitud se hace en una comida preparada por la familia del «actor-bailarín». La mayoría de los «actores» son jóvenes entre los 15 y 25 años, aunque también participan señores de hasta 41 años, como es el caso del señor Virgilio López Aguilar quien lleva diez años participando en pastorelas y dos o tres en el Juego de la Conquista. Las ocupaciones de cada uno de los integrantes varían, desde es- tudiantes de informática hasta conductores de camiones escolares o cerrajeros. Cuando el equipo de trabajo está integrado, el tiempo de ensayos oscila entre treinta y sesenta días, en los que se reúnen en la casa del director o los mayordomos para asignar personajes y aprender los diálogos.

El primer día de la

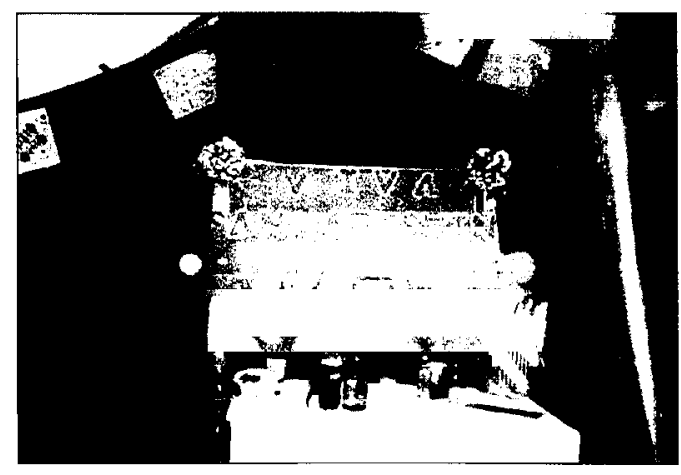

Capilla para los festejos de San Agustín Tlacotepec. representación, los ejecutantes se preparan a partir de las 9 de la mañana; se trasladan al lugar que funge como camerino, ahí comienzan a cambiarse de ropa; en esos momentos el nerviosismo se amalgama con una atmósfera de burlas y risas al verse por primera vez con el vestuario y accesorios que portarán durante el día. Mientras esto sucede, en el escenario en que se llevará a cabo la danza el director supervisa que todo se encuentre en orden y en su lugar. El juego se realiza a un costado del kiosco en el centro del pueblo; en el lugar se observa una explanada en la que se encuentra una plataforma hecha de madera, la cual funge como el único elemento escenográfico de la Danza, ya que como ha explicado Arango,

...en México, el culto sagrado se verifica al aire libre. [...] Las representaciones cristianas permanecen al aire libre, siguen siendo culto indígena en forma cristiana como todo lo que pertenece a él, respecto a procesiones, arcos de flores y enramadas, altares, música y bailes» ${ }^{4}$.

A las 11 horas, da inicio el juego de la conquista con un baile en el que se presenta el bando de los aztecas; luego de 20 minutos aproximadamente hacen su aparición los españoles, quienes llegan en una camioneta que simboliza un barco. A partir de ese momento se narran los episodios más relevantes de la conquista de México-Tencochtitlan. El segundo día culmina con el tormento a Cuauhtémoc y la muerte de Moctezuma, hechos que enmarcan el fin del mundo azteca. Posteriormente se efectúa un baile popular en el que participa la mayoría de los habitantes del pueblo.

Durante estos dos días de juego, en el pueblo de San Agustín se vive un ambiente de fiesta en el que se involucra toda la comunidad. Por un lado, los danzantes, quienes ejecutan las acciones del drama con una devoción extrema, llegando incluso hasta lesionarse de
1

Lilian Scheffler, Juegos tradicionales del Estado de Tlaxcala, México, SEP, 1976, pág. 89.

2

Carlo Bonfiglioli y Jesús Jáuregui, Las danzas de conquista 1 . México contemporáneo, México, FCE, 1996, pág. 22.

3

José Luis González explica sobre los mayordomos: «La otra gran institución popular ligada al desarrollo del culto católico y de las fiestas ha sido el sistema de mayordomías. Esta institución, aunque tiene variantes de un contexto a otro, en todas partes conserva como característica principal el ser una estructura jerarquizada de cargos en orden de asegurar las celebraciones festivas de la comunidad" ("El catolicismo popular y su aporte a la configuración de la cultura mexicana», en Guillermo Bonfil Batalla, comp., Simbiosis de culturas, México, F.C.E., 1993, pág. 552).

4

Manuel Antonio Arango, El teatro religioso en la América Hispana, Barcelona, Puvill Libros S.A., pág. 154

El juego de la conquista de San Agustín Tlacotepec (Oaxaca. México)

KARINA CAS'IRO SAN'JANA 


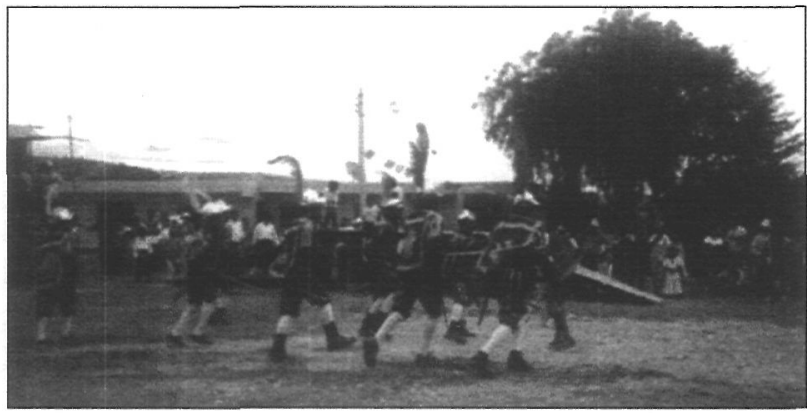

Soldados españoles en el Juego de la Conquista de San Agustín Tlacotepec. gravedad con los machetes que portan para la ejecución. Por otro lado, el resto de los habitantes de la comunidad, que participan como espectadores, quienes paulatinamente a lo largo de la representación abandonan su postura pasiva y terminan por integrarse al juego con comentarios, risas e interviniendo también en los diálogos y los bailables, ya que, como dice Guiraud en relación con la Danza y el juego: «Los espectáculos (y la danza puede ser considerada como tal) son, a la vez juegos y artes: juegos desde el punto de vista de los actores, artes desde el punto de vista de los espectadores ${ }^{5}$.

Organizar y participar en la fiesta es tomar conciencia y compartir el orgullo de ser tlacotepense. Se participa, quizás como ya ocurría antes de la llegada de los europeos, en una ceremonia que permite expresar el sentido de colectividad y trabajo grupal de una comunidad:

Los ritos nahuas poseen una multitud de elementos teatrales. Es fácil echar a volar la imaginación y tratar de visualizar la escenografía, las danzas, el vestuario, el maquillaje, la utilería, la acción, e imaginar la música y los cantos. En el papel de actores y espectadores participan todos los estratos de la sociedad náhuatl, por lo que se debe también conocer cómo estaba estructurada y qué funciones se ejercían dentro de la mismá6.

Actualmente se sigue considerando característico de los pueblos, el trabajo comunitario:

La comunalidad es la gran lección que podemos ofrecer a la sociedad, de ahí que la veamos con gusto reproducirse en la ciudad (Oaxaca), en las colonias, en los barrios pobres, incluso en las grandes ciudades como el D.F. o Los Ángeles, California, en donde encontramos representantes auténticos de esta resistencia heroica, actual y legítima, que dibuja la imagen contemporánea de nuestros pueblos autóctonos oaxaqueños?.

En San Agustín Tlacotepec, se observa El juego de la conquista de San Agustín Tlacotepec (Oaxaca. México) KARINA CASTRO SANTANA. que el resto del año cada quien se dedique a ocupaciones diversas, porque la oportunidad de regresar al pueblo natal en la fiesta anual no se puede perder, debido a que también es la ocasión para reencontrarse con amigos y familiares que regularmente no se frecuentan. Como explica José Luis González,

La fiesta anual ha venido siendo, hasta nuestros días, hasta el gran ritual colectivo en el que no sólo las estancias y los ranchos circundantes, sino también los que han emigrado más lejos, se encuentran en el pueblo para participar en una fiesta en la que se reconocen como miembros de una colectividad8.

Las danzas de conquista encuentran también su origen en el carácter ritual con el que los naturales abordaban los hechos trascendentales en una comunidad, es decir, la repercusión idiosincrásica, religiosa y política que regulaba su vida cotidiana.

Cuando los españoles llegaron a tierras americanas buscaron la forma de convertir a los naturales al catolicismo; sin embargo los obstáculos como la diferencia de lenguas, comportamientos y costumbres, obstruyeron la comunicación para iniciar una evangelización efectiva9. Los recursos visuales resultaron ser convenientes para garantizar una explicación de la doctrina católica:

... el primer grupo de misioneros tomó el teatro como elemento didáctico para la enseñanza evangelizadora. Se representaron obras en lengua náhuatl, donde los indios eran actores, cantantes y al mismo tiempo bailaban en los escenarios. Los misioneros dirigían las obras [e] iban induciendo rápidamente al cristianismo ${ }^{10}$.

De esta manera el teatro y las representaciones dancísticas-teatrales resultaron ser los procedimientos más recurridos por los frailes y catequizadores europeos, incluyendo elementos de parafernalia muy vistosos que se utilizaban desde antiguo en los rituales prehispánicos. En la siguiente cita, se observa la descripción detallada de una celebración narrada por fray Bernardino de Sahagún, en la cual se distingue, sobre todo, la parafernalia y el vestuario colorido que utilizaban los antiguos mexicanos en sus ceremonias rituales de sacrificios:

Al séptimo mes llamaban tecuilhuitontli. En este mes hacían fiesta y sacrificio a la diosa de la sal que se llamaba Uixtocíhuatl [...] Los atavíos de esta diosa eran de color amarillo, y una mitra con muchos plumajes 
verdes que salían de ella, como penachos altos [...] tenía en las gargantas de los pies atados cascabeles de oro, o caracolitos blancos, estaban ingeridos en una tira de cuero de tigre; cuando andaba hacían gran sonido [...] llevaba en la mano un bastón rollizo y en lo alto como un palmo o dos ancho, como paleta, adornado con papeles goteado de ulli, tres flores hechas de papel, una en cada tercio; las flores de papel iban llenas de incienso, junto a las flores iban unas plumas de quetzalli cruzadas, o aspadas; cuando bailaba en el areito íbase arrimando al bastón y alzándole al compás del baile ${ }^{11}$.

A propósito de estas manifestaciones prehispánicas que se consideran como un posible teatro, María Sten expone lo siguiente:

...se desprende claramente la existencia de los espectáculos palaciegos y populares, religiosos y profanos, la existencia de lugares especiales para las representaciones, escuelas para los cantores y danzantes, ensayos previos al espectáculo, el uso de máscaras pelucas, pinturas faciales, elementos de escenografía, el diversificado carácter de las danzas y de los cantos, elementos cirqueros y burlescos [...]. Pero este teatro rudimentario que cuenta ya con todos los elementos de la representación tradicional, tiene algo más que lo distingue de modo especial; es un [...] teatro-espejo del hombre y de su mundo; espectáculo religioso que al mismo tiempo es una fuente inapreciable para los estudios antropológicos, que permite descubrir la filosofía de aquella sociedad, sus mitos, el Olimpo de sus dioses, su cosmología, su astrología, su magia y sus ritos ${ }^{12}$.

De esta manera se observa que el sincretismo que se produjo a partir de la conquista de México prevalece hasta nuestros días en las manifestaciones culturales mexicanas; sobre todo las que se generan en comunidades indígenas:

El teatro del Nuevo Mundo tiene un marcado influjo indígena en el estilo, en el colorido y quizá en algunas formas del indio. La decoración de los escenarios en la Nueva España y Perú, corresponde al ritual mágico, indígena. El disfraz, en su mayor parte es indígena. El elemento musical y baile tienen raíces rituales del mundo indio ${ }^{13}$.

En la actualidad, las danzas de conquista obtienen un significado en el que los habitantes de cada comunidad viven el conflicto de diversas maneras. Más que cumplir una función de evangelizar o educar a los pueblos, las danzas se ejecutan con diversas intenciones entre las que destacan la recreación y búsqueda de

la identidad comunitaria:

Hablar de «fiesta» es, antes que nada, referirnos a una comunidad que se reconoce, hacia dentro y hacia fuera, compartiendo un sentido que en primera instancia, ha tenido una referencia

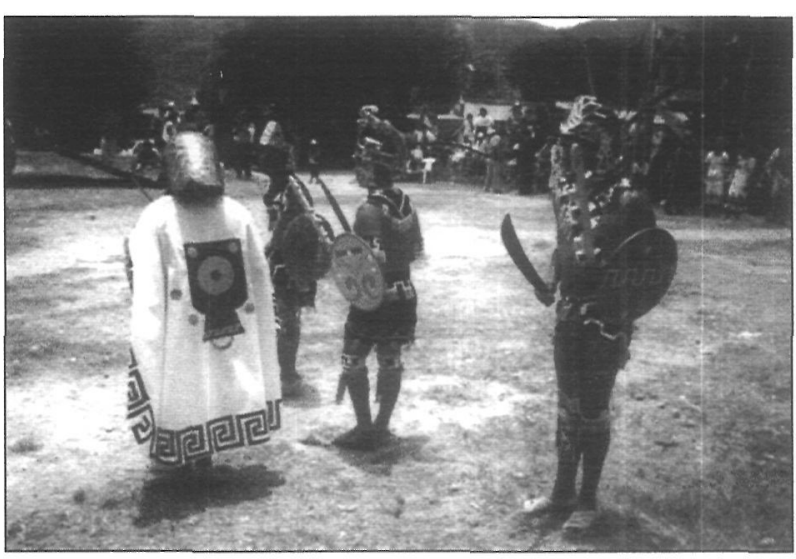

Indígenas en el Juego de la Conquista San Agustín Tlacotepec. religiosa. Navidad, Miércoles de ceniza, Cuaresma, Semana Santa, Corpus Christi, el Día de muertos, la Virgen de Guadalupe, los santuarios, las fiestas patronales de cada pueblo, etc., son otros tantos ejes jerarquizados para la organización del tiempo familiar, comunal, regional y nacional [...]; son la sistematización de la memoria colectiva popular tejida de mitos, ritos y «costumbres» ${ }^{14}$.

Debido entre otros factores al arraigo de sus tradiciones, el pueblo mexicano sigue ejecutando actualmente gran parte de sus danzas, como una forma de asegurar y recrear su identidad cultural. En el caso específico de San Agustín Tlacotepec, la importancia y repercusión de su danza de conquista radica en un alto grado de identificación con los motivos de lucha de la parte indígena y en que la victoria asegurada por el bando de los españoles no es vivida como algo negativo, sino como lo que justifica o antecede a la aceptación de la liturgia católica, elemento fundamental actual para explicar y entender el sentido de las acciones, festejos y determinaciones que sustentan igualmente la estructura social comunitaria. Un dato curioso, que es digno de mencionarse, es una ocasión en la que uno de los directores asignados llamado Fulgencio Guzmán tuvo la ocurrencia de incluir un estandarte de la Virgen de Guadalupe en el bando de los aztecas con la intención de alentar la fe católica en sus paisanos; sin embargo tal decisión generó diversas opiniones al respecto entre los tlacotepenses, argumentando algunos que era una arbitrariedad utilizar la imagen de la Guadalupana en un contexto en el que cronológicamente es imposible situar, mientras que otros apoyaban la idea, por considerar que era un recurso efectivo para continuar fomentando la religiosidad en la comunidad. De hecho, así ocurre en otras representaciones de la conquista como la que
11

Bernardino de Sahagún, Historia General de las cosas de la Nueva España, México, Porrúa, 1997, pág. 119.

\section{2}

María Sten, Vida y muerte del teatro nahuatl; el Olimpo sin Prometeo, México, SEP, 1974, págs. 30-31.

13

Arango, op. cit., pág. 157.

14

Ibidem, pág. 547
El juego de la conquista de San Agustín Tlacotepec (Oaxaca. México)

KARINA CASTRO SANTANA 


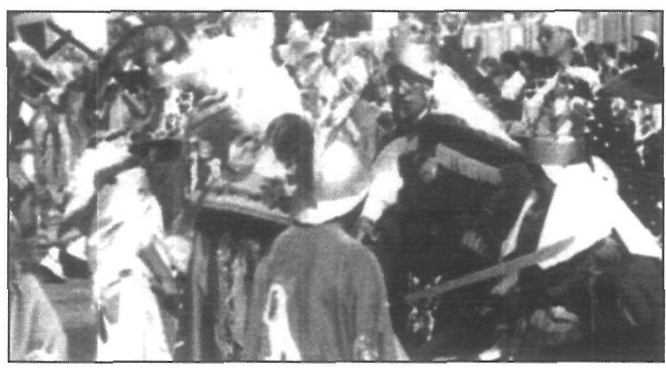

Desarrollo del Juego de la Conquista.

15

Juventino Castro, Diálogo de mestizos, antiensayo sobre 10 mexicano, México, ABISA, 1997, pág. 32

16

Augusto Boal, Técnicas Latinoamericanas de Teatro Popular, México, 1982, pág. 21

17

Cit. en Castro, op. cit., págs. 33 y 34 .

18

Boal, op. cit., pág. 70 .

19

Llamado así por su presentación «informal» en cuanto a carencia de un edificio teatral se refiere, así como por el estilo tan peculiar de vincularse los participantes de la manifestación artística con los habitantes que fungen como público.

20

Peter Brook, El espacio vacío, arte y técnicas del teatro, Barcelona, 1972, pág. 85.

El juego de la conquista de San Agustín Tlacotepec (Oaxaca. México)

KARINA CASTRO SANTANA tiene lugar dentro de las celebraciones guadalupanas del 12 de diciembre, la cual ofrecen los habitantes de Amecameca, un pueblo que se ubica en el Estado de México: ellos se trasladan en peregrinación hasta la ciudad de México para ejecutar la danza frente al atrio de la Basílica de Guadalupe, para la cual, como explica Juventino Castro, «hasta la fecha los indígenas se visten con sus plumas y sus atuendos [...] el día que se celebra a la virgen de Guadalupe, o cualquier otra fecha católicopagana, y danzan como lo hacían sus ancestros, en el Tepeyac u otros lugares sagrados desde antiguo» ${ }^{15}$.

Retomando la representación en San Agustín, el hecho de integrar la danza dentro de la fiesta patronal constituye un acto de regulación de las relaciones sociales en la comunidad, es decir, la preparación y participación en la fiesta funcionan de manera semejante a los rituales católicos actualmente practicados (bautizos, confirmaciones, primera comunión, etc.).

Reconociendo en la danza-drama de Tlacotepec una participación colectiva, se puede discurrir que se trata de teatro popular. En primer lugar, cabe aclarar que el término popular se aplica a los espectáculos que se llevan a cabo por el pueblo. A continuación se expone la propuesta de Augusto Boal, quien define el teatro popular, a partir de dos conceptos: población y pueblo.

Población es la totalidad de habitantes de un país o región. Más restringido es el concepto de "pueblo»: incluye sólo a quienes alquilan su fuerza de trabajo. Pueblo es una designación genérica que engloba a obreros, campesinos y a todos los que están temporaria u ocasionalmente asociados a los primeros, como ocurre con los estudiantes y otros sectores en algunos países. Quienes constituyen la población pero no el pueblo -o sea los anti-pueblo- son los propietarios, los latifundistas, la burguesía y sus asociados (ejecutivos, mayordomos) y, en general, todos los que piensan como ellos ${ }^{16}$.

Boal ofrece además una categorización del teatro popular en la que se ubica la representación de San Agustín Tlacotepec, atendiendo sus particularidades:

Teatro del pueblo y para el pueblo: en esta categoría se habla de un trabajo realizado por el pueblo estan- do él mismo como destinatario, es decir, la creación colectiva a partir de las ideas del pueblo, quienes pretenden plantear alguna solución ante una circunstancia para mejorar su comunidad o simplemente para cumplir un ciclo, como es el ejemplo de la danza de conquista de San Agustín Tlacotepec, la cual se celebra cada año en el marco de la fiesta en honor al santo patrono para fomentar la unión entre los habitantes de esta comunidad. Esta categoría de teatro del pueblo y para el pueblo se divide en tres formas del teatro las cuales tienen diversos objetivos.

a) De propaganda: Con un tinte político, este tipo de teatro funciona como un medio de denuncia ante ciertas situaciones que enfrentan las clases bajas ante las altas.

b) Didáctico: Esta forma de teatro tiene como objetivo proponer una enseñanza para el pueblo, se tratan temas comunes allegados a su vida cotidiana para comprender mejor su entorno.

c) Cultural: El teatro cultural, busca la diversión y el hecho de rescatar tradiciones como danzas, carnavales, etcétera ${ }^{17}$.

De acuerdo a la clasificación empleada por Boal, se identifica que la representación analizada contiene las características del «Teatro del pueblo y para el pueblo» en la categoría «cultural», ya que, como se ha mencionado, el juego tiene por objetivo principal divertir y reunir una vez al año a los habitantes de la comunidad. En palabras de Boal,

Esta es una de las formas teatrales más simples, en las que el pueblo manifiesta libremente sus ideas y emociones. Es, de todas las formas teatrales, la más 'colectiva' la que más inspira y estimula el trabajo conjunto $^{18}$.

Para complementar la definición de teatro popular, cabe citar a Peter Brook, quien habla de un "teatro tosco» ${ }^{19}$, el cual define como: «el teatro que no está en el teatro, el teatro en carretas, en carromatos, en tablados, con el público que permanece en pie, bebiendo, sentado alrededor de las mesas de la taberna, incorporado a la representación, respondiendo a los actores» 20 .

Es evidente que el teatro popular tiene su origen en el planteamiento de una necesidad de una comunidad que mantiene vivas sus tradiciones como una forma de confirmación y divulgación de su identidad.

Considerando que la Danza, como ceremonia de culto a San Agustín, muestra una forma ritual, es pertinente citar los llamados «ritos de paso», de los que se ofrece una breve descripción, para después ubicar la danza 
celebrada en San Agustín Tlacotepec, según lo estipulado en los diferentes ritos expuestos:

A diferencia de los ritos del ciclo de vida, tienen como principal función marcar el paso del tiempo para los grupos sociales y el paso de dichos grupos por el tiempo. [...] En general se trata de grandes celebraciones comunales que indican cambios estacionales 0 anuales y señalan para determinado grupo su tránsito de un estado de normalidad previo relativamente estable y fijo, a otro posterior. [...] Como todas las secuencias ceremoniales que señalan el tránsito de una situación social a otra, constan de tres momentos principales. Se inician con los rituales de separación de la condición social normal que conducen a los ritos liminales y constituyen el estado de marginación o de intemporalidad social, para concluir con los ritos de agregación por los cuales se retorna a la situación normal, pero en un nuevo estado ${ }^{21}$.

La estructura de la fiesta indica el carácter ritual de la danza, planteando fases específicas por las que transcurre la representación como rito. Se habla de tres períodos: uno preliminal, un período liminal y otro postliminal. Todos ellos constituyen los ritos de separación e incorporación, es decir, la vuelta a la armonía o flujo de vida cotidiana en la comunidad antes y después de los días de fiesta.

En términos más descriptivos, el período preliminal consiste en las acciones preparatorias de vestir los trajes apropiados, el traslado al área donde se ejecutará la danza y un intermedio para comer el primer día. El período liminal se caracteriza por el desarrollo del conflicto ritual en el tiempo extracotidiano por medio de la danza teatro. Finalmente, el último período marca una nueva situación de vuelta a la armonía en el tiempo real.

A continuación se clasifican en los periodos descritos anteriormente, la estructura de la Danza y la transición de la comunidad durante la representación:

(DANZA) P. Preliminal: Mundo azteca, unidad armónica. P. Liminal: Confrontación entre españoles y aztecas. P. Posliminal: Fundación de la Nueva España, nueva unidad armónica. (COMUNIDAD) P. Preliminar: Preparativos, Ensayos, Organización, Vestuario. P. Liminal: Representación durante dos días. P. Posliminal: Incorporación a la vida cotidiana y confirmación de su identidad.

Se debe hacer hincapié en que la representación se lleva a cabo sin ninguna condición económica, es decir, todas las personas que participan en el juego lo hacen por una cuestión religiosa, por pagar una manda $u$ ofreci- miento que hayan hecho a cambio de algún «milagro» solicitado a San Agustín. Incluso, algunos habitantes de Tlacotepec deben trasladarse desde diversos lugares para participar en la fiesta, debido a que la mayoría han emigrado a la ciudad de México o a los Estados Unidos de Norteamérica.

En conclusión, la danza de conquista es una tradición en Tlacotepec, dado que la comunidad se niega a romper con un ritual que los mantiene unidos, por lo menos dos o tres meses al año. Más que venerar por medio de su fraternidad y unión a su Santo Patrono San Agustín, la fiesta constituye una oportunidad de recrear una serie de acciones que conforman su identidad. De hecho, como explica Elio Masferrer, ya «en el siglo XIX los historiadores están trabajando los movimientos indígenas desde una perspectiva que incluye un papel de la religión como elemento movilizador» ${ }^{22}$.

Cabe destacar que desde que llegaron las danzas de conquista a territorios mexicanos, éstas se han constituido en más que una tradición. Se trata de un medio de expresión para las comunidades indígenas que aún desean mostrar su cultura y su punto de vista respecto a un hecho que fue trascendental en la historia de México, así como asegurar y preservar una identidad colectiva. En cualquier caso, es evidente que la religión católica continúa siendo un factor fundamental en la vida de las comunidades indígenas y mestizas de nuestro país, en el que las prácticas rituales cuentan con una vigencia, gracias al respeto y devoción con que prevalecen las tradiciones mexicanas que se han fortalecido a través de todos estos siglos.

\section{BIBLIOGRAFÍA}

Azor, Ileana, Teatralidades y carnaval. Danzantes y color en Puebla de los Ángeles, México, Gestos, Universidad de las Américas, 2004.

Arango, Manuel Antonio, El teatro religioso colonial en la América bispanoamericana, Barcelona, Puvill Libros, S.A.

Boal, Augusto, Técnicas latinoamericanas de teatro popular, México, Nueva Imagen, 1982.

Bonfiglioli, Carlo, Fariseos y matachines en la Sierra Tarabumara: entre la pasión de Cristo, la transgresión cómico-sexual y las danzas de conquista, México, Instituto Nacional Indigenista, 1993.
21

Bonfiglioli y Jáuregui, op. cit., pág. 22.

22

Elio Masferrer Kan, «Movimientos indios y cuestión religiosa. Encuentros y desencuentros con la antropología en México", en Warman, op, cit., pág. 279. 
- y Jesús Jáuregui, Las danzas de conquista I. México contemporáneo, México, Fondo de Cultura Económica, 1996.

Bonfil Batalla, Guillermo (compilador), Los inmigrantes y su cultura en México, México, Fondo de Cultura Económica, 1993.

-, Culturas populares y política cultural, México, Consejo Nacional para la Cultura y las Artes, 1995.

Brook, Peter, El espacio vacio, arte y técnicas del teatro, Barcelona, Península, 1972.

Castro, Juventino, Diálogo de mestizos, México, Abastecedora de Impresos Abisa, 1992.

Díaz del Castillo, Bernal, Historia verdadera de la conquista de la Nueva España, México, Porrúa, 1996.
Fossier, Robert, La Edad Media. vol. 2: El despertar de Europa, Barcelona, Grijalbo, 1988.

Sahagún, Bernardino de, Historia general de las cosas de la Nueva España, México, Porrúa, 1997.

López Austin, Alfredo, El pasado indígena, México, F.C.E., 1996.

Scheffler, Lilian, Juegos tradicionales del estado de Tlaxcala, México, SEP, 1976.

Toríz, Martha, La fiesta prebispánica: un espectáculo teatral, México, INBA, 1993.

Warman, Arturo y Arturo Argueta (coordinadores), Movimientos indigenas contemporáneos en México, México, CIIH (Centro de Investigaciones Interdisciplinarias en Humanidades), Universidad Nacional Autónoma de México, 1993. 